\title{
A Class III Semaphorin (Sema3e) Inhibits Mouse Osteoblast Migration and Decreases Osteoclast Formation In Vitro
}

\author{
Alun Hughes · Jennifer Kleine-Albers • \\ Miep H. Helfrich • Stuart H. Ralston • \\ Michael J. Rogers
}

Received: 12 August 2011/Accepted: 4 December 2011/Published online: 7 January 2012

(C) The Author(s) 2012. This article is published with open access at Springerlink.com

\begin{abstract}
Originally identified as axonal guidance cues, semaphorins are expressed throughout many different tissues and regulate numerous non-neuronal processes. We demonstrate that most class III semaphorins are expressed in mouse osteoblasts and are differentially regulated by cell growth and differentiation: Sema3d expression is increased and Sema3e expression decreased during proliferation in culture, while expression of Sema3a is unaffected by cell density but increases in cultures of mineralizing osteoblasts. Expression of Sema3a, $-3 \mathrm{e}$, and $-3 \mathrm{~d}$ is also differentially regulated by osteogenic stimuli; inhibition of GSK3 $\beta$ decreased expression of Sema3a and $-3 e$, while $1,25-(\mathrm{OH})_{2} \mathrm{D}_{3}$ increased expression of Sema3e. Parathyroid hormone had no effect on expression of Sema3a, -3b, or $-3 \mathrm{~d}$. Osteoblasts, macrophages, and osteoclasts express the Sema3e receptor PlexinD1, suggesting an autocrine and paracrine role for Sema3e. No effects of recombinant Sema3e on osteoblast proliferation, differentiation, or mineralization were observed; but Sema3e did inhibit the migration of osteoblasts in a wound-healing assay. The formation of multinucleated, tartrate-resistant acid phosphatase-positive osteoclasts was decreased by $81 \%$ in cultures of mouse bone marrow macrophages incubated
\end{abstract}

A. Hughes $(\bowtie) \cdot$ J. Kleine-Albers · M. H. Helfrich $(\bowtie)$.

M. J. Rogers

Musculoskeletal Research Programme, School of Medicine \& Dentistry, University of Aberdeen, Institute of Medical Sciences,

Foresterhill, Aberdeen AB252ZD, UK

e-mail: mmd403@yahoo.co.uk

M. H. Helfrich

e-mail: m.helfrich@abdn.ac.uk

S. H. Ralston

Molecular Medicine Centre, Western General Hospital,

University of Edinburgh, Crewe Road, Edinburgh EH42XU, UK with $200 \mathrm{ng} / \mathrm{mL}$ Sema3e. Correspondingly, decreased expression of osteoclast markers (Itgb3, Acp5, Cd51, Nfatc1, CalcR, and Ctsk) was observed by qPCR in macrophage cultures differentiated in the presence of Sema3e. Our results demonstrate that class III semaphorins are expressed by osteoblasts and differentially regulated by differentiation, mineralization, and osteogenic stimuli. Sema3e is a novel inhibitor of osteoclast formation in vitro and may play a role in maintaining local bone homeostasis, potentially acting as a coupling factor between osteoclasts and osteoblasts.

Keywords Macrophage $\cdot$ Plexin $\cdot$ Mineralization · Vitamin $\mathrm{D}_{3} \cdot$ Migration

Semaphorins are signaling molecules, originally described as axonal guidance molecules [1], consisting of eight classes, of which five (classes III-VII) are widely expressed in mammalian tissues [2,3]. Of these five classes, all are membrane-tethered except for the secreted class III semaphorins, which consist of seven members (Sema3a-g) that display $35-52 \%$ protein homology [3]. Similar to other semaphorins, the class III semaphorins have a sema domain that interacts with binding domains of receptor complexes consisting of a plexin (usually of the PlexinA class) and, in the case of the class III semaphorins alone, a neuropilin (Npn-1 or -2) [3]. Only Sema3e is capable of binding to a plexin alone (although notably to PlexinD1, not PlexinA) without the requirement of a neuropilin [4].

Little is known about the role of the class III semaphorins in the skeleton, although several studies have described expression of some of the family members and their related receptors. Expression of Sema3a [5, 6], Sema3b [7], Npn-1 [6, 8], PlexinA1 [6], PlexinA2 [6], and 
PlexinD1 [9] has been observed in both primary and cultured osteoblasts. Expression of Npn-1 was not detected in osteocytes, suggesting that Npn-1 expression is downregulated during osteoblast differentiation into osteocytes [8]. Sema3a, Npn-1, PlexinA1, and PlexinA2 expression has been reported in chondrocytes, while only expression of the receptor components was observed in osteoclasts [6]. Sema3e has been observed in areas of differentiating bone structures in the mouse embryo [10], although the precise cell types expressing this protein have not been elucidated.

Here, we describe the expression of class III semaphorins and several of their receptors in primary mouse calvarial osteoblasts. We demonstrate that expression of Sema3a, $-3 \mathrm{~d}$, and $-3 \mathrm{e}$ is differentially affected by cell growth or differentiation, $1,25(\mathrm{OH})_{2} \mathrm{D}_{3}$, and pharmacological inhibition of GSK3 $\beta$. Our data confirm that Sema3e is produced by osteoblasts, may act in an autocrine fashion to inhibit osteoblast migration, and may act as a paracrine regulator of osteoclasts due to the inhibitory effects of recombinant Sema3e on osteoclast formation.

\section{Materials and Methods}

\section{Reagents}

$\alpha$-Minimum essential medium ( $\alpha$-MEM), fetal calf serum, (FCS), penicillin, and streptomycin were from Invitrogen (Paisley, UK). Tissue culture plates were from Costar (Cambridge, MA). All other reagents were from Sigma (Poole, UK), unless otherwise indicated.

\section{Mouse Calvarial Osteoblast Cultures}

Osteoblasts were isolated from the calvaria of 4-day-old litters of C57B16 mice by sequential collagenase/EDTA digestion. Osteoblasts were cultured in $\alpha$-MEM (10\% FCS, $100 \mathrm{U} / \mathrm{mL}$ penicillin, $100 \mathrm{mg} / \mathrm{mL}$ streptomycin, and $1 \mathrm{mM}$ glutamine) and grown to $\sim 90 \%$ confluence. Cells were harvested in trypsin/EDTA, counted on a Neubauer hemocytometer, seeded at $3 \times 10^{4}$ cells $/ \mathrm{cm}^{2}$, and allowed to adhere overnight prior to treatment. Mineralization was induced using $\alpha$-MEM supplemented with $10 \%(\mathrm{v} / \mathrm{v})$ FCS, $100 \mathrm{U} / \mathrm{mL}$ penicillin, $100 \mathrm{mg} / \mathrm{mL}$ streptomycin, $1 \mathrm{mM}$ glutamine, $10 \mathrm{mM} \beta$-glycerophosphate, and $50 \mu \mathrm{g} / \mathrm{mL}$ L-ascorbic acid 2-phosphate. Medium was replaced every 3 days. Mineralization was visualized by staining with alizarin red on ethanol-fixed monolayers.

Generation of Mouse Osteoclasts from Bone Marrow

Mouse osteoclasts were generated in vitro from macrophage colony-stimulating factor (M-CSF)-dependent bone marrow macrophages. Bone marrow cells were flushed from the tibiae and femora of 3- to 4-week-old C57B16 mice and cultured overnight in $75-\mathrm{cm}^{2}$ tissue culture flasks (one flask per mouse; BD Biosciences, San Jose, CA) in $\alpha$-MEM containing $100 \mathrm{U} / \mathrm{mL}$ penicillin, $100 \mu \mathrm{g} / \mathrm{mL}$ streptomycin, $1 \mathrm{mM}$ glutamine, $10 \% \mathrm{FCS}$, and $25 \mathrm{ng} / \mathrm{mL}$ murine M-CSF (R\&D Systems, Minneapolis, MN). Nonadherent cells were removed, pelleted, and counted before being seeded at $1 \times 10^{5}$ cells $/ \mathrm{cm}^{2}$ in $75-\mathrm{cm}^{2}$ tissue culture flasks in the presence of $25 \mathrm{ng} / \mathrm{mL}$ M-CSF. Medium was changed and fresh M-CSF added every 2 days until confluence, after which cells were trypsinized, counted, and seeded at $5 \times 10^{4}$ cells $/ \mathrm{cm}^{2}$ in $\alpha$-MEM containing $100 \mathrm{U} /$ $\mathrm{mL}$ penicillin, $100 \mu \mathrm{g} / \mathrm{mL}$ streptomycin, $1 \mathrm{mM}$ glutamine, $10 \%$ FCS, $50 \mathrm{ng} / \mathrm{mL}$ murine M-CSF, and $10 \mathrm{ng} / \mathrm{mL}$ murine RANKL (R\&D Systems). Fresh medium and cytokines were added every 2 days until multinucleated osteoclasts formed (approximately 5 days).

\section{Isolation and Culture of Mature Rabbit Osteoclasts}

Mature osteoclasts were isolated from the limbs of New Zealand white rabbits, 2-4 days old, as described previously [11]. Osteoclasts isolated from the minced long bones of one rabbit were vortexed and resuspended in $25 \mathrm{~mL}$ of $\alpha$-MEM containing $100 \mathrm{U} / \mathrm{mL}$ penicillin, $100 \mu \mathrm{g} /$ $\mathrm{mL}$ streptomycin, $1 \mathrm{mM}$ glutamine, and $10 \%$ FCS. Cells were seeded in 125-uL aliquots onto 5-mm-diamter dentine discs before being allowed to adhere for $2 \mathrm{~h}$. Nonadherent cells were washed away in PBS, before incubation in the presence or absence of recombinant Sema3e for $48 \mathrm{~h}$. Cells were then fixed in $4 \%$ formaldehyde, stained with TRITCphalloidin, and counted for the presence of actin rings by inverted fluorescence microscopy. Discs were then stained for tartrate-resistant acid phosphatase (TRAP) as previously described [12], and TRAP-positive multinucleated cells with three or more nuclei were considered to be osteoclasts. Cells were cleaned from the discs, and the area of resorption pits on the surface of the dentine was quantified by reflected light microscopy and custom image analysis software developed using Aphelion ActiveX objects (ADCIS, Herouville-Saint-Clair, France).

\section{Extraction of Total RNA}

RNA was extracted by phenol/chloroform fractionation using TRIzol ${ }^{\circledR}$ reagent (Invitrogen) per the manufacturer's instructions. Isolated RNA was further purified using an RNeasy Mini Kit (Qiagen, Valencia City, CA), including a DNAse I treatment step. RNA quantity was determined by spectrophotometry, and $\mathrm{A}_{260} / \mathrm{A}_{280}$ ratios were used to confirm the absence of contamination. RNA quality was confirmed on an Agilent (Palo Alto, CA) Bioanalyzer to ensure 
that no degradation occurred during extraction. RNA was reverse-transcribed to cDNA using $2 \mu \mathrm{g}$ of total RNA, $1 \mu \mathrm{g}$ of random primer oligonucleotides (Invitrogen), and MMLV reverse transcriptase (SuperScript II, Invitrogen).

\section{End-Point RT-PCR Analysis of Gene Expression}

Intron-spanning oligonucleotide primers were designed using Primer3 [13] and checked via BLAST for specificity (http://blast.ncbi.nlm.nih.gov/Blast): Sema3e forward, 5'CCA TAC AAT GCT GCT GGA TG-3'; Sema3e reverse, 5'-GTG TGC CAA TTA TAT CCG GG-3'; PlexinD1 forward, 5'-TCA GAG GAG ATC GTG TGT GC-3'; PlexinD1 reverse, $5^{\prime}$-GCT CTA TGC TCC AGG TCC TG$3^{\prime}$; Gapdh forward, 5'-ACT TTG TCA AGC TCA TTT CC-3'; Gapdh reverse, 5'-TGC AGC GAA CTT TAT TGA TG-3'. PCRs were prepared to a total volume of $25 \mu \mathrm{L}$ containing the equivalent of $50 \mathrm{ng}$ of reverse-transcribed RNA per reaction and $500 \mathrm{nM}$ of each primer. Amplification was performed using Qiagen Taq DNA polymerase under optimized conditions: $95^{\circ} \mathrm{C}$ for $10 \mathrm{~min}, 33$ cycles of $95^{\circ} \mathrm{C}$ for $30 \mathrm{~s}, 58^{\circ} \mathrm{C}$ for $60 \mathrm{~s}$, and $72^{\circ} \mathrm{C}$ for $60 \mathrm{~s}$. Products were resolved on a $1.5 \%$ agarose gel and detected using ethidium bromide under UV illumination.

\section{Quantitative PCR Analysis of Gene Expression}

Intron-spanning, FAM-labeled hydrolysis probe assays were designed for genes of interest using the Universal Probe Library (Roche, Mannheim, Germany; see Table 1) and multiplexed with a primer-limited, VIC-labeled Gapdh TaqMan $^{\circledR}$ assay (Applied Biosystems, Foster City, CA). Assays were performed on a LightCycler $^{\circledR} 480$ qPCR system (Roche) using LightCycler Probes Mix and an optimized protocol $\left(20 \mu \mathrm{L}\right.$ reaction volume; $5 \min 95^{\circ} \mathrm{C}$, 40 cycles of $10 \mathrm{~s} 95^{\circ} \mathrm{C}, 30 \mathrm{~s} 60^{\circ} \mathrm{C}$ ). Relative gene-ofinterest expression to Gapdh was calculated using a standard curve of serially diluted cDNA to correct for PCR efficiency, per the method of Pfaffl [14].

\section{Western Blot Analysis of Sema3e Protein}

Protein lysates were prepared in RIPA buffer $(1 \%[\mathrm{v} / \mathrm{v}]$ Nonidet P-40, $0.1 \%$ [w/v] sodium dodecyl sulfate, $0.5 \%$ [w/ v] sodium deoxycholate in PBS, plus $1 \%[\mathrm{v} / \mathrm{v}]$ protease inhibitor cocktail [Sigma]). Cell lysate $(40 \mu \mathrm{g})$ was electrophoresed on $12 \%$ polyacrylamide-SDS gels (Criterion XP system; Bio-Rad, Hemel Hempstead, UK) under reducing conditions. Proteins were transferred to polyvinylidene difluoride membranes and then hybridized with $0.2 \mu \mathrm{g} / \mathrm{mL}$ anti-Sema3E antibody (R\&D Systems), followed by $1 \mu \mathrm{g} /$ $\mathrm{mL}$ anti-goat immunoglobulin G-horseradish peroxidise conjugate (Merck, Darmstadt, Germany). Bands were visualized using chemiluminescence (Supersignal West Dura reagent; Pierce, Rockford, IL) and a Bio-Rad Fluor-S Max MultiImager.

Measurement of Alkaline Phosphatase Activity

Alkaline phosphatase activity was measured by the conversion of $p$-nitrophenyl phosphate to $p$-nitrophenyl. Osteoblasts were cultured on 96-well plates and lysed in $150 \mu \mathrm{L}$ of lysis buffer (1 M diethanolamine [DEA], $1 \mathrm{mM}$ $\mathrm{MgCl}_{2}$, and $0.05 \%$ Triton $\left.\mathrm{X}-100\right)$. Lysate $(50 \mu \mathrm{L})$ was mixed with $50 \mu \mathrm{L}$ of substrate solution $(20 \mathrm{mM} p$-nitrophenol phosphate, $1 \mathrm{M} \mathrm{DEA}$, and $1 \mathrm{mM} \mathrm{MgCl}_{2}$ ). The amount of $p$-nitrophenol generated after $30 \mathrm{~min}$ was calculated by measuring the absorption of each well at $414 \mathrm{~nm}$ in comparison with a standard curve of $1.25-30 \mathrm{nmol} /$ well.

\section{Scratch Wound Assay}

A $0.5-\mathrm{mm}$ wound was made, using a pipette tip, across a confluent monolayer of osteoblasts on 24-well Imagelock plates (Essen Instruments, Ann Arbor, MI). Cell layers were washed three times, treated with $40 \mathrm{nM}$ PTH or $100 \mathrm{ng} / \mathrm{mL}$ recombinant Sema3e in medium with or without $10 \%(\mathrm{v} / \mathrm{v})$ serum, incubated in an IncuCyte microscope (Essen Instruments), and imaged every $30 \mathrm{~min}$. Wound healing was calculated using the IncuCyte Scratch Wound Assay software application. Results were confirmed using replicate plates and images taken on a phase-contrast inverted light microscope.

\section{Statistical Analysis}

One-way analysis of variance (ANOVA) followed by a (Bonferroni/Dunnett's) post hoc test was used to statistically analyze results using SPSS version 15.0 software (SPSS, Inc., Chicago, IL). $P \leq 0.05$ was considered statistically significant.

\section{Results}

Expression of Class III Semaphorins in Mouse Calvarial Osteoblasts

Using a panel of quantitative PCR (qPCR) assays to measure all seven known members of the class III semaphorins, we examined their expression profile in both proliferating ( $\mathrm{P}$, after 48-hour growth in normal medium) or mineralizing (M, after 14 additional days in osteoblast differentiation medium) C57B16 calvarial osteoblasts (Fig. 1a). Six of the seven class III semaphorins were detected in osteoblasts (the 
Table 1 Universal Probe Library assays for quantitative PCR

\begin{tabular}{llll}
\hline Gene & Forward primer & Reverse primer & UPL probe \\
\hline Sema3a & 5'-ATC AGT GGG TGC CTT ACC AA & 5'-GCC AAA TGT TTT ACT GGG ACA & 72 \\
Sema3b & 5'-CTT CGG CTC TCC TTT CAA GA & 5'-CAA GGC TTC ATA ACA GCA GGT & 19 \\
Sema3c & 5'-ATG GCC ACT CTT GCT CTA GG & 5'-CAT CTT GTC TTC GGC TCC TC & 48 \\
Sema3d & 5'-GGA AAA GCG ACA AGA GTT GC & 5'-TGA AAA TTT TGT TTT TCA AAC ACT G & 4 \\
Sema3e & 5'-GGG GCA GAT GTC CTT TTG A & 5'-AGT CCA GCA AAC AGC TCA TTC & 63 \\
Sema3f & 5'-GAA GGA GGA ACG CGG AAG & 5'-AGG CAG TGA CAA GCA TCG T & 38 \\
Plexinal & 5'-CGC CAA GTC CAA GGA CGA G & 5'-CTG CCT TCG AGC CTG TTC & 21 \\
Plexind1 & 5'-CTG GAT GTC CAT CTG CAT GT & 5'-CAG GAA GAA CGG CTC ACC TA &
\end{tabular}

exception being Sema3f), as was expression of the receptor components PlexinA1 and -D1. Sema3c was the most abundant, with low levels of Sema3b, $-3 \mathrm{~d}$, and $-3 \mathrm{~g}$ detected in proliferating osteoblasts. Fourteen days treatment in differentiation medium resulted in mineralization (as confirmed by alizarin red staining) (data not shown) and a significant increase in expression of Sema3a, $-3 \mathrm{~d}$, and $-3 \mathrm{~g}$ as well as PlexinD1 (by 2.2-, 14.6-, 6.5-, and 4.6-fold, respectively). Sema3e significantly decreased by $90 \%$ in mineralizing cultures, while Sema3c remained unchanged and Sema3f remained undetected. We chose three of the significantly regulated genes for further investigation, choosing Sema3a, $-3 \mathrm{~d}$, and $-3 \mathrm{e}$ based on reports of each displaying effects on cells or in tissues unrelated to axonal guidance.

Long culture periods ( $>1$ week) in the presence of differentiation medium are required to produce cultures of mineralizing osteoblasts. In order to discount the effects of culture time or cell density on the expression of key members of the class III semaphorins, we examined expression of Sema3a, $-3 \mathrm{~d}$, and $-3 \mathrm{e}$ in osteoblasts seeded at a low density and grown toward confluence in normal, nondifferentiation medium (Fig. 1b). Osteoblasts proliferated until confluence at 96-120 h postseeding and remained viable during the time course. Expression of Sema3a remained unchanged, while $3 \mathrm{~d}$ significantly increased in expression to 11.2-fold over control by $216 \mathrm{~h}$. Sema3e significantly decreased in expression as cells grew to confluence, reaching a fivefold decrease by $216 \mathrm{~h}$. After reaching confluence, expression of both Sema3d and -3e stabilized. These observations suggest that the changes in expression of Sema3d and -3e observed in cultures of proliferating and mineralizing cultures were related to the effects of cell growth and density and that the increase in Sema3a expression in mineralizing cultures was not related to cell growth or density.

Sema3a, -3d, and -3e Are Differentially Regulated by Osteogenic Factors

Next, we examined the expression of Sema3a, $-3 \mathrm{~d}$, and $-3 \mathrm{e}$ in response to factors known to influence osteoblast differentiation. PTH may act as both an anabolic and a catabolic factor in bone, depending on the timing and duration of exposure [15]. Short-term exposure of calvarial osteoblasts to PTH induced an elevation of alkaline phosphatase activity (data not shown), indicating that osteoblast differentiation had been initiated [16]. Despite this, we did not see an effect on the expression of Sema3a, $-3 \mathrm{~d}$, or $-3 \mathrm{e}$ after 24-hour exposure (Fig. $2 \mathrm{a}-\mathrm{c}$ ) or at earlier or later time points (data not shown) following PTH stimulation. Proliferation and differentiation of osteoblasts in vitro can also be induced by 1,25-dihydroxyvitamin $\mathrm{D}_{3}\left(1,25[\mathrm{OH}]_{2} \mathrm{D}_{3}\right)$ treatment $[17,18]$ and has previously been reported to regulate Sema3b expression in osteoblasts [7]. In contrast to the inert effects of PTH, $1,25(\mathrm{OH})_{2} \mathrm{D}_{3}$ caused a significant increase in expression of Sema3e after 24 h (Fig. 2c), which persisted for at least $48 \mathrm{~h}$ posttreatment (data not shown) but had no effect on Sema3a or 3d expression (Fig. 2a, b). Stimulation of the canonical Wnt signaling pathway promotes the translocation of $\beta$-catenin to the nucleus and the subsequent regulation of gene transcription via binding to TCF/LEF. Wnt signaling is important in the development and maintenance of mineralized tissues [19] and has previously been implicated in the regulation of Sem3d in zebrafish [20] and Sema3a in the dental mesenchyme [21] but has not been investigated in osteoblasts in vitro. Downstream stimulation of the canonical Wnt pathway can be achieved by pharmacological inhibition of GSK $\beta$ (which phosphorylates $\beta$-catenin to target it for ubiquitin-mediated degradation [22]) by 6-bromoindirubin-3'-oxime (BIO) [23]. Treatment with MeBIO, the kinase inactive negative control of $\mathrm{BIO}$, had no significant effect on expression of Sema3a, $-3 \mathrm{~d}$, or $-3 \mathrm{e}$, while BIO caused a significant decrease in expression of both Sema3a and -3e (Fig. 2a, c). Cells were also treated with $40 \mathrm{mM} \mathrm{LiCl}$, which inhibits $\mathrm{GSK} \beta$ but via a different mechanism from BIO. $\mathrm{LiCl}$ inhibition of $\mathrm{GSK} \beta$ also caused a decrease in Sema3a and $-3 d$ expression, supporting the data observed with BIO (data not shown). These data demonstrate that there is evidence of 
Fig. 1 Differential expression of semaphorin class III members in proliferating or mineralizing mouse osteoblasts. a Calvarial osteoblasts were treated during proliferation (P) or mineralizing state $(\mathrm{M}$, after 14 days in $\beta$-glycerophosphate- and ascorbic acid-supplemented medium) and harvested for RNA to generate cDNA. Quantitative PCR was performed to measure expression of class III semaphorins and the two major plexins involved in their receptor complexes, PlexinA1 and PlexinD1. Results are expressed as relative units normalized to Gapdh expression. b Osteoblasts were seeded at $3 \times 10^{4}$ cells $/ \mathrm{cm}^{2}$ and allowed to proliferate in standard $\alpha$-MEM. Cells were harvested as indicated, and quantitative PCR was performed for Sema3a, Sema3d, and Sema3e and displayed as fold change from $0 \mathrm{~h}$. Results are from three independent experiments with triplicate technical replicates. ${ }^{*} p<0.05$, $* * p<0.01, * * * p<0.001$

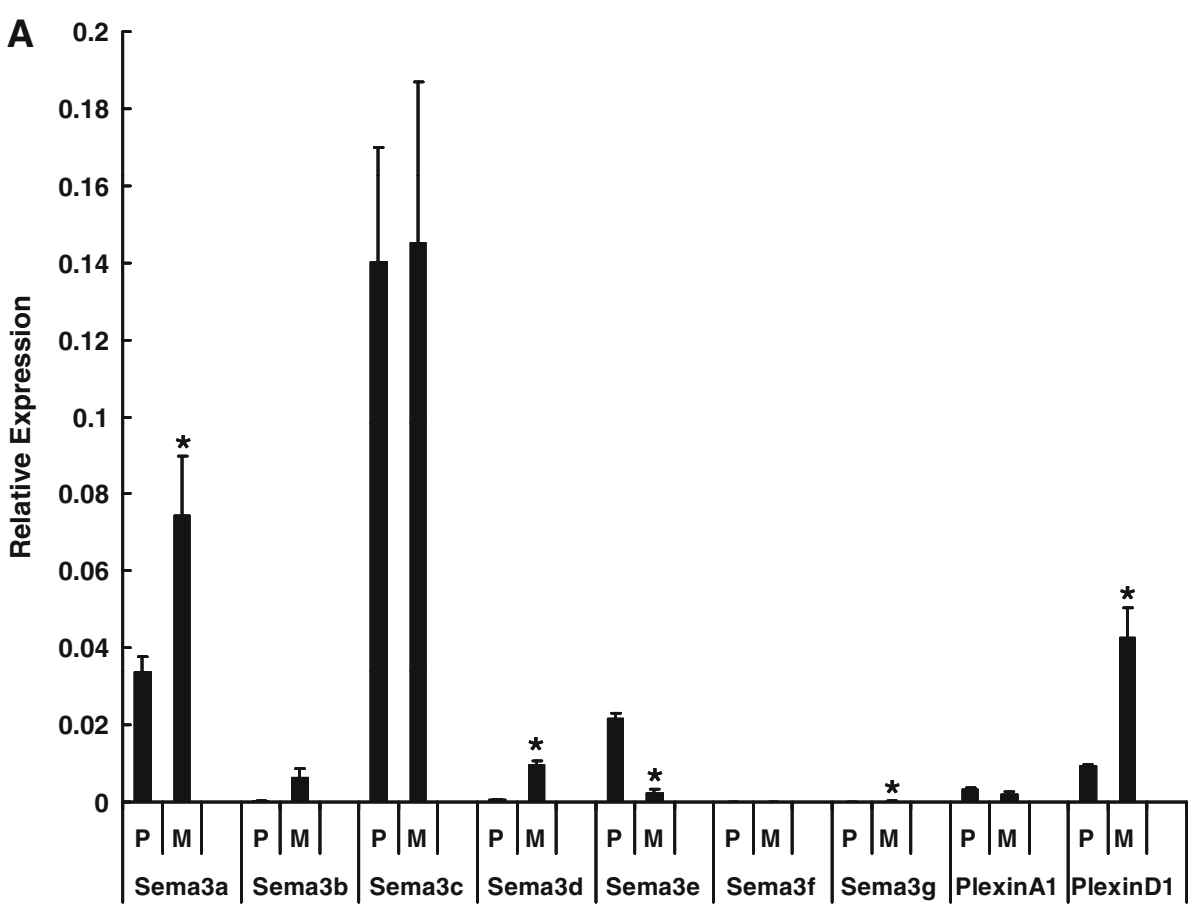

B

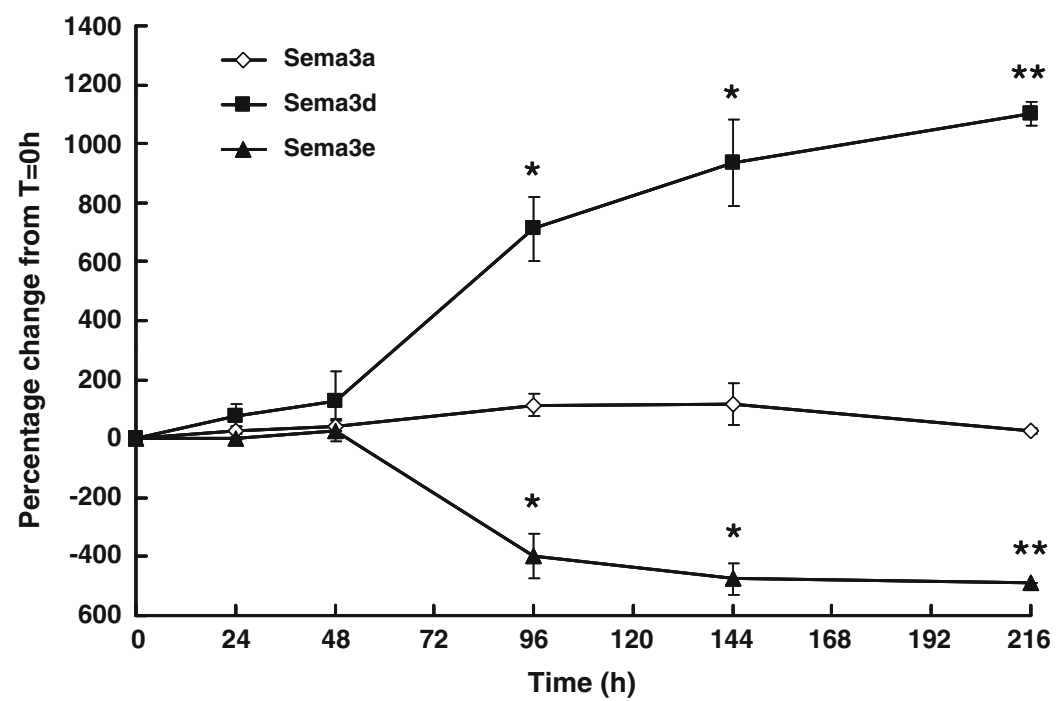

differential regulation of class III semaphorins in response to modulation of signaling pathways known to be important for osteoblast function.

\section{Expression of Sema3e and Its Associated Receptor, PlexinD1, in Bone Cells}

As Sema3b has recently been reported to be a $1,25(\mathrm{OH})_{2} \mathrm{D}_{3}$-responsive gene in osteoblasts [7], we decided to focus on the similarly $1,25(\mathrm{OH})_{2} \mathrm{D}_{3}$-responsive Sema3e for further study. Sema3e is a unique class III semaphorin as it does not require the presence of a neuropilin in its receptor complex, instead consisting solely of
PlexinD1. Since class III semaphorins can antagonize VEGF signaling by competing for neuropilins, Sema3e will not exhibit non-Plexin-mediated effects associated with neuropilin sequestration, thereby avoiding potential interpretation problems due to the expression of VEGF receptors in osteoblasts (reviewed in [24]). Sema3e expression was strongly detected in osteoblasts by both end-point and qPCR but below the limit of detection in macrophages and osteoclasts (Fig. 3a, b). Sema3e protein was clearly detected in both proliferating and mineralizing osteoblast cultures but not osteoclasts (Fig. 3d). Trace amounts of Sema3e protein were detected in macrophage cultures, possibly representing some heterogeneity within the cell 

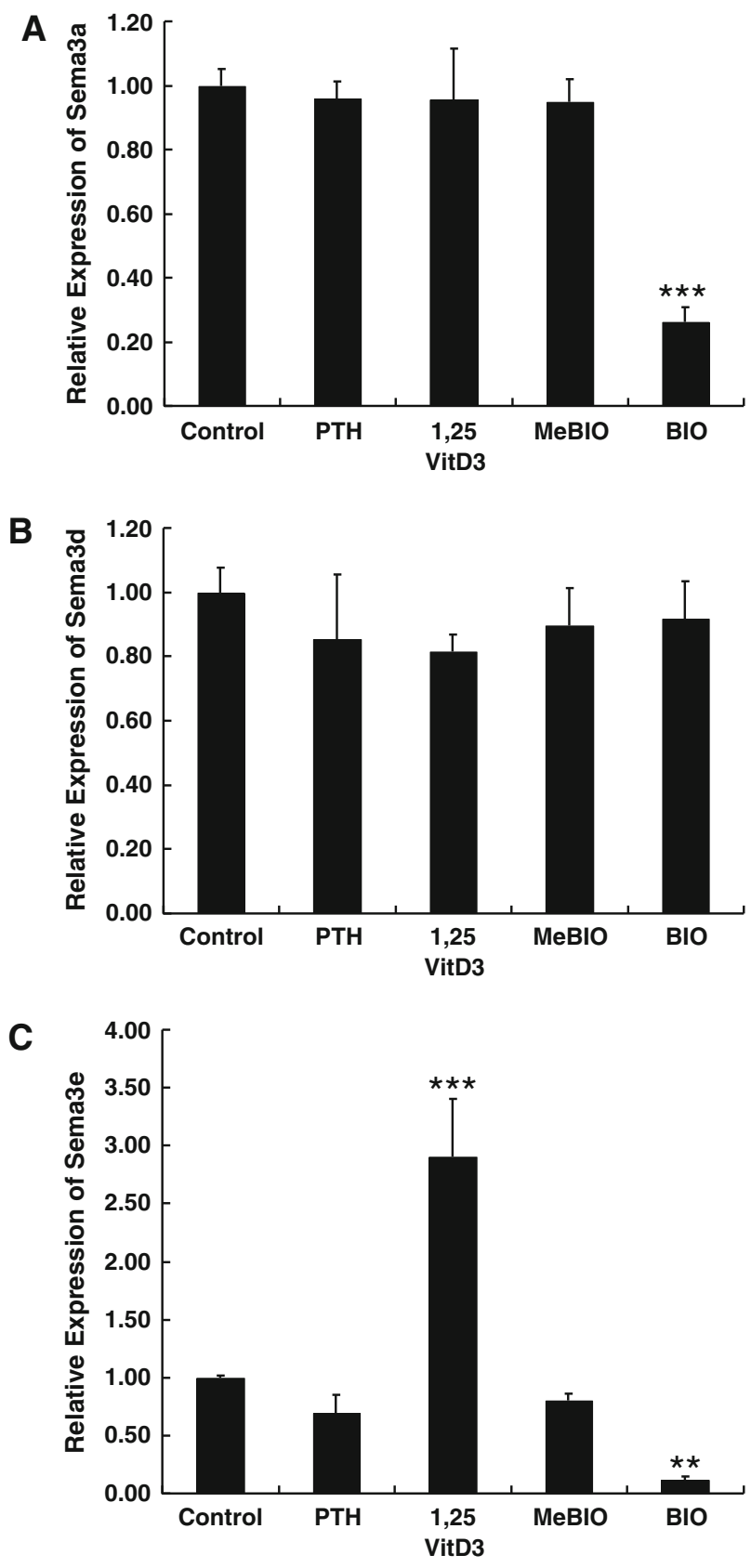

Fig. 2 Expression of Sema3a, Sema3d, and Sema3a in response to parathyroid hormone, 1,25-dihydroxyvitamin $\mathrm{D}_{3}$, lithium chloride, or the GSK3 inhibitor BIO. Osteoblasts were seeded at $3 \times 10^{4}$ cells/ $\mathrm{cm}^{2}$ and treated with $40 \mathrm{nM} \mathrm{PTH}, 10 \mathrm{nM} 1,25-(\mathrm{OH})_{2} \mathrm{D}_{3}, 40 \mathrm{mM} \mathrm{LiCl}$, $5 \mu \mathrm{M}$ of $\mathrm{BIO}$, or the kinase-inactive control MEBIO. Quantitative PCR was performed upon cDNA generated from total RNA, and expression of a Sema3a, b Sema3d, or c Sema3e was normalized to Gapdh. Results are from three independent experiments with triplicate technical replicates and are displayed as relative expression calibrated to control samples. ${ }^{*} p<0.05, * * p<0.01,{ }^{* * *} p<0.001$

population. Higher protein levels were observed in cultures of mineralizing osteoblasts compared to proliferating osteoblasts, despite the lower transcript expression observed in mineralizing cultures, although this may be due
A

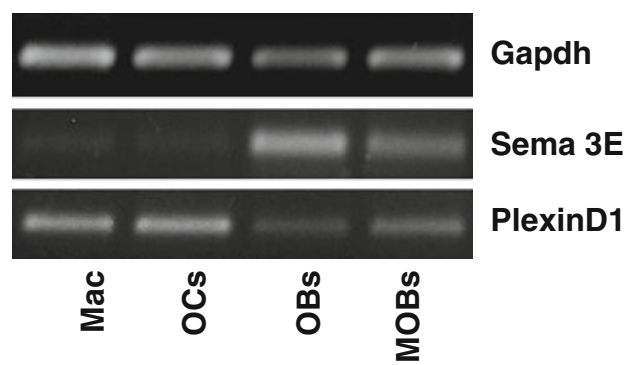

B

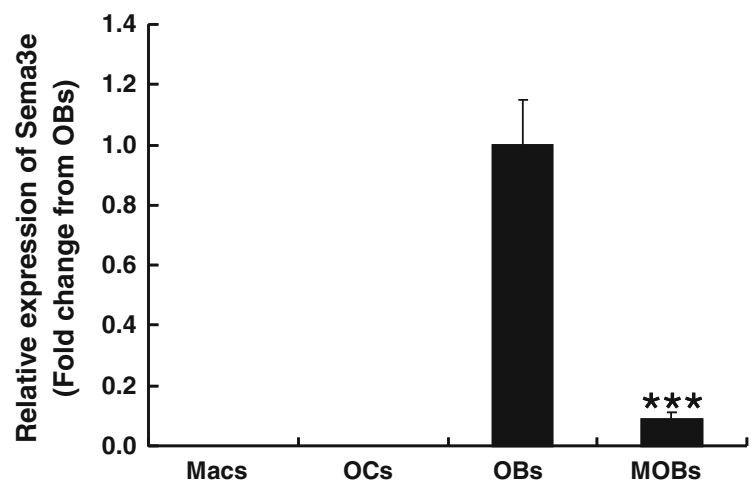

C

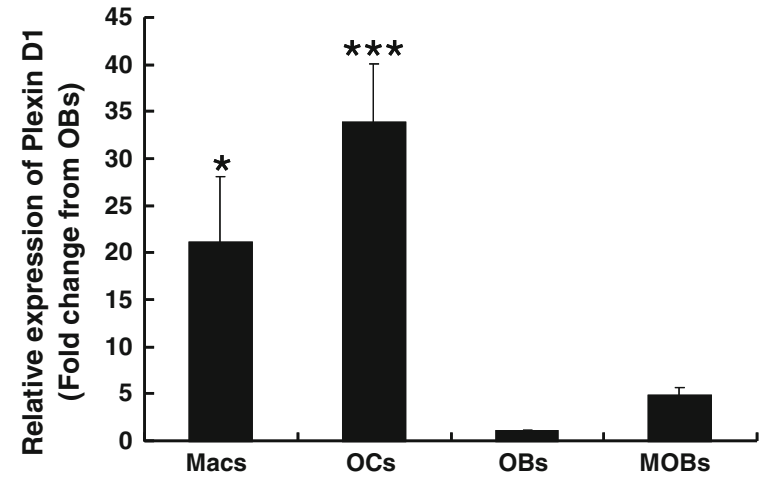

D

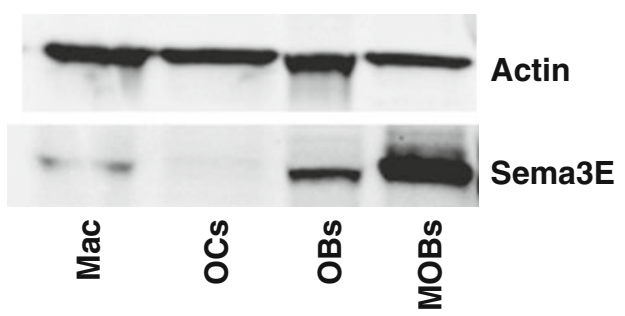

Fig. 3 Expression of Sema3e and its receptor PlexinD1 in mouse bone cells. Mouse macrophages (Macs), osteoclasts $(O C s)$, proliferating calvarial osteoblasts $(O B s)$, and mineralizing calvarial osteoblasts (MOBs, 14 days in $\beta$-glycerophosphate- and ascorbic acidsupplemented medium) were harvested for protein and RNA. a End-point PCR showing Sema3e and PlexinD1 expression in all four cell types. Quantitative-PCR showing relative abundance of $\mathbf{b}$ Sema3e or c PlexinD1 in bone cells in vitro, both normalized to Gapdh and calibrated to the expression observed in OBs. d Western blot of Sema3E protein expression in lysates generated from identical samples used in PCR analysis. Representative blots are from three independent experiments $(\mathbf{a}, \mathbf{d})$ and from three independent experiments with triplicate technical replicates (b, c). ${ }^{*} p<0.05$; $* * p<0.01, * * * p<0.001$ 
to deposition and accumulation of secreted Sema3e in the extracellular matrix. All cell types examined (osteoblasts, M-CSF-dependent macrophages, and osteoclasts) expressed the Sema3e receptor protein PlexinD1 (Fig. 3a). Expression of PlexinD1 was significantly higher in macrophages and osteoclasts than in either osteoblast culture (Fig. 3c). As expression of PlexinD1 was detected in all cells, this suggests that osteoblast-derived Sema3e may act as both an autocrine signaling molecule and in a paracrine manner upon macrophages and/or osteoclasts. We found no change in expression of PlexinD1 after treatment with $1,25(\mathrm{OH})_{2} \mathrm{D}_{3}, \mathrm{BIO}$, or PTH (data not shown) and encountered problems detecting PlexinD1 protein levels in mouse cells using available antibodies.

Recombinant Sema3e Decreases the Migration of Osteoblasts

Since osteoblasts expressed both Sema3e and PlexinD1, we investigated Sema3e as an autocrine regulator of osteoblast function in vitro. Recombinant mouse Sema3e (100 ng/ $\mathrm{mL}$ ) did not affect osteoblast proliferation, measured by alamar blue assay of viable cell number (data not shown), nor did it affect mineralization, measured by alizarin red staining after 7, 14, or 21 days in culture (21-day representative image shown in Fig. 4a). PTH (40 nM) caused differentiation of osteoblasts and a subsequent doubling in alkaline phosphatase activity after 48-hour treatment. Sema3e $(100 \mathrm{ng} / \mathrm{mL})$ did not affect alkaline phosphatase activity in the presence or absence of PTH (Fig. 4b) but did inhibit the migration of osteoblasts in a wound-healing assay using a confluent monolayer of osteoblasts. PTH increased wound healing, and serum-free medium resulted in no migration of cells into the wound area (Fig. 4c). As wound closure can occur as a result of both migration and proliferation, it was important to establish that the effects observed in Fig. $4 \mathrm{c}$ were predominantly due to migration. By constructing time-lapse videos from the still images taken for calculating wound width, we could confirm that wound closure occurred due to migration of cells across the wounded region and not due to proliferation of cells along the wound edges (data not shown).

Recombinant Sema3e Decreases the Formation of Osteoclasts

As macrophages and osteoclasts express PlexinD1, we next investigated the effect of $200 \mathrm{ng} / \mathrm{mL}$ recombinant mouse Sema3e protein on the formation of multinucleated (three or more nuclei per cell), TRAP-positive osteoclasts in M-CSF- and RANKL-treated cultures of mouse bone marrow macrophages. An abundance of TRAP-positive osteoclast-like cells was observed in control cultures after
A
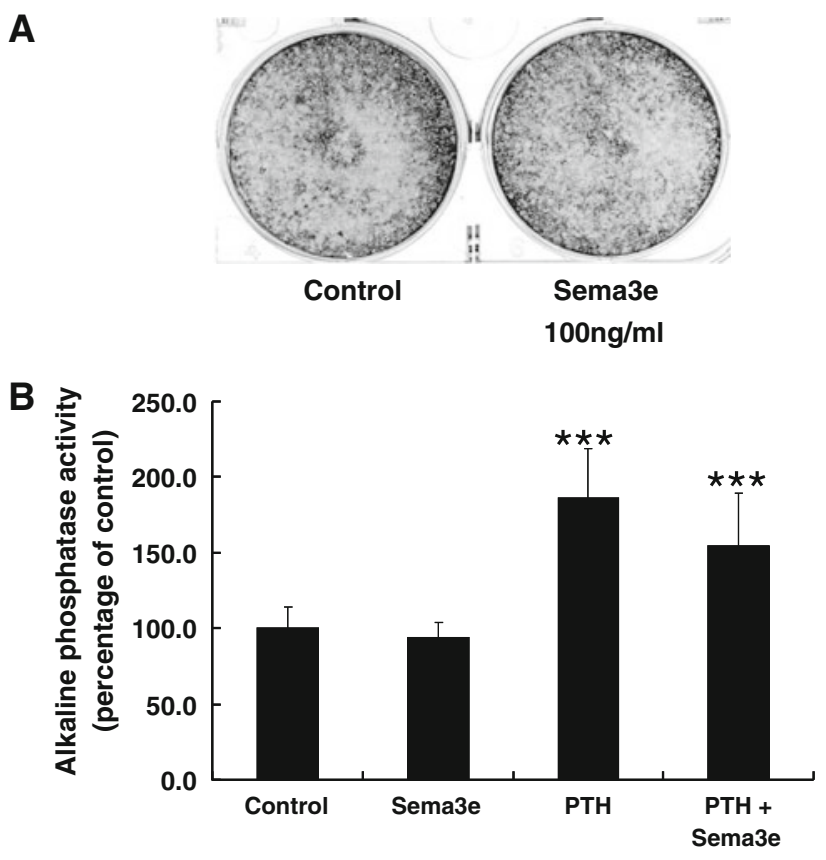

C

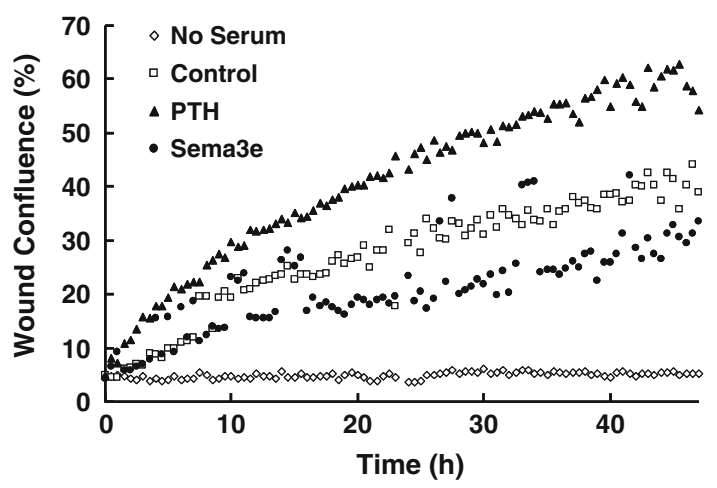

Fig. 4 Effects of recombinant Sema3e protein on mineralization, differentiation, and wound healing of mouse calvarial osteoblasts. a Mouse calvarial osteoblasts were treated for up to 21 days in the presence of $\beta$-glycerophosphate- and ascorbic acid-supplemented medium with or without $100 \mathrm{ng} / \mathrm{mL}$ recombinant mouse Sema3e, fixed, and stained with alizarin red. b Alkaline phosphatase activity of calvarial osteoblasts was measured after 48 -hour exposure to $40 \mathrm{nM}$ PTH with or without $100 \mathrm{ng} / \mathrm{mL}$ Sema3e. c Wound closure (as a percent of wound confluence) was measured every $30 \mathrm{~min}$ on an IncuCyte microscope system following treatment with medium containing no serum (diamond), medium plus serum (square), medium plus serum and $40 \mathrm{nM}$ PTH (filled triangle), or medium plus serum and $100 \mathrm{ng} / \mathrm{mL}$ Sema3e (dot). Representative images from three independent experiments (a), three independent experiments with triplicate technical replicates (b), and one experiment with four replicates $(\mathbf{c}) . * p<0.05, * * p<0.01, * * * p<0.001$

approximately 5 days in RANKL, while Sema3e-treated cultures had fewer osteoclasts and less intense TRAP staining (Fig. 5a), which was confirmed by Western blotting for TRAP protein (Fig. 5b). Quantification of osteoclasts revealed that Sema3e-treated cultures had $62 \%$ fewer osteoclasts than control cultures (Fig. 5c), with a dosedependent decrease in osteoclast number observed from 50 
Fig. 5 Effects of recombinant Sema3e on osteoclast formation and expression of markers of differentiation. Mouse osteoclasts formed from M-CSF-dependent mouse marrow macrophages treated with $50 \mathrm{ng} / \mathrm{mL}$ murine M-CSF and $10 \mathrm{ng} / \mathrm{mL}$ murine RANKL $\pm 200 \mathrm{ng} / \mathrm{mL}$ recombinant mouse $\mathrm{Sema}$ e for on average, 5 days. a Cultures were imaged at $10 \times$ in phase contrast (upper panels) or stained for TRAP and imaged at $20 \times$ (lower panels). b Western blot for TRAP from protein lysates. c Quantification of total number of osteoclasts TRAP $^{+}$ and three or more nuclei per cell) per well on a 96-well plate. d Gene expression profiling by qPCR of osteoclast markers. Images are representative of five independent experiments (a, b) and of three independent experiments with 10 replicates per experiment (c) and with triplicate technical replicates per experiment (d). ${ }^{*} p<0.05$, $* * p<0.01, * * * p<0.001$
A
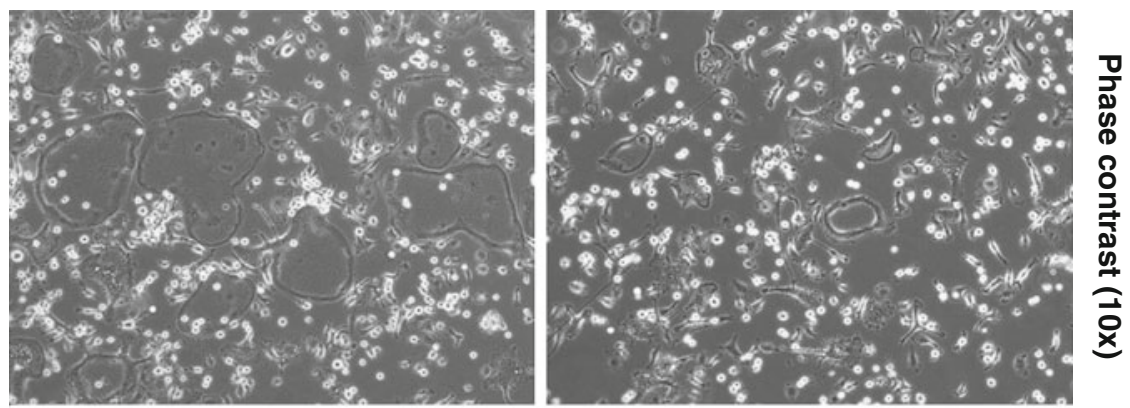

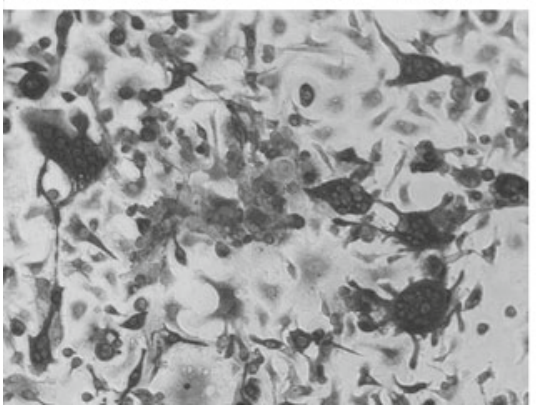

Control

B

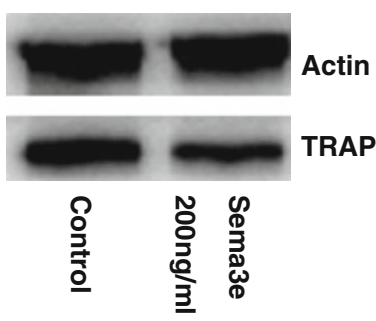

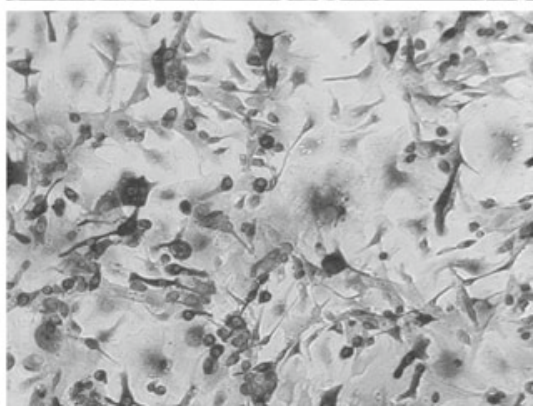

Sema3e 200ng/ml

\section{$\frac{1}{8}$

D

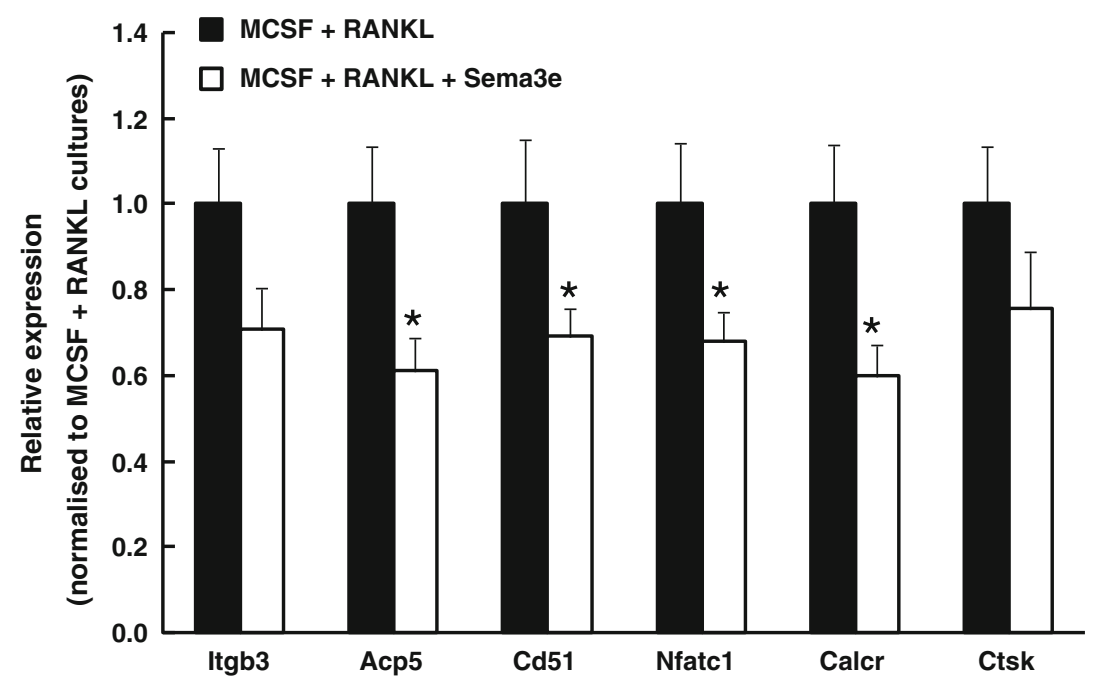


A

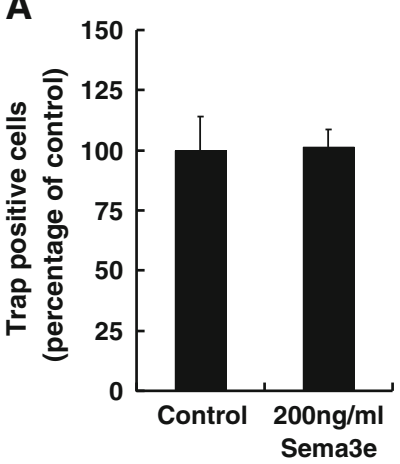

B

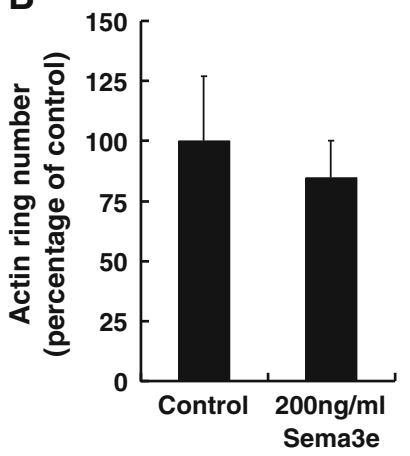

C

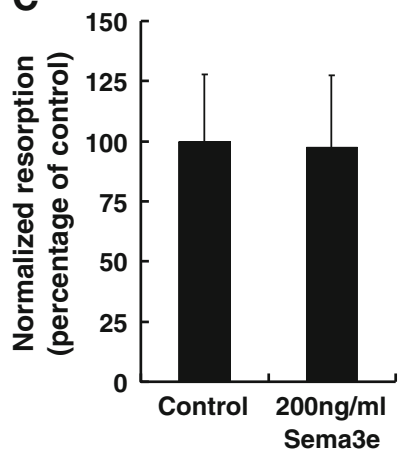

Fig. 6 Effects of recombinant Sema3e on mature rabbit osteoclast resorption. Mature rabbit osteoclasts were isolated from the long bones of neonatal rabbits allowed to attach on dentine discs, then treated with or without $200 \mathrm{ng} / \mathrm{mL}$ recombinant Sema3e for $48 \mathrm{~h}$. a Osteoclast number was determined by TRAP staining $\left(\mathrm{TRAP}^{+}\right.$and

(data not shown). In order to ascertain if Sema3e could affect osteoclast function as well as formation, we investigated the effects of Sema3e treatment on mature osteoclast cultures. As mature osteoclast cultures from mice formed variably on dentine, we used mature osteoclasts isolated from the minced long bones of rabbit neonates to remove errors associated with varying formation efficiencies or the inhibitory effects of Sema3e on formation. The use of mature rabbit osteoclasts on dentine is an established method for the measurement of resorption, enabling the easy identification of TRAP-positive osteoclasts and functional actin rings. No effect of Sema3e was observed on the number of TRAP-positive multinucleated cells adhered to the dentine surface, the formation of actin rings, or the area of dentine resorbed by mature osteoclasts (Fig. 6a-c).

\section{Discussion}

Functional roles independent of axonal guidance have been found for numerous semaphorins, and class III members have been implicated in vascular patterning [4], angiogenesis ([25], reviewed by [26]), immune responses including T-cell activation ([27], reviewed by [28]), and the regulation (both positive and negative) of cancer cells ([10, 25, 29, 30], reviewed by [31]). Some of these roles may be attributable to antagonism of VEGF signaling due to competition for neuropilins, which are coreceptors for both VEGF and most class III semaphorin receptor complexes [32-34]. As the receptor complex for Sema3e consists only of PlexinD1 and does not require a neuropilin cofactor, any pleiotropic effects of Sema3e cannot be due to interference with VEGF signaling [4].

Wnt signaling is an important mediator of osteoblast differentiation and can signal either through canonical three or more nuclei per cell). b Actin rings were quantified by phalloidin staining for actin. c Total resorbed area of dentine was normalized to the number of $\mathrm{TRAP}^{+}$cells adhered to each disc. Results are from two independent experiments with eight replicates per experiment

( $\beta$-catenin-dependent) or noncanonical ( $\beta$-catenin-independent) pathways. Sema3a is positively regulated by Wnt4 in the dental mesenchyme [21], although it is unclear if this is via canonical or noncanonical Wnt signaling. Sema3d has been suggested to be downstream of Wnt signaling via TCF in zebrafish neural crest cells, indicating a possible role of canonical Wnt signaling in the positive regulation of Sema3d [22]. Contrary to what has been observed in the dental mesenchyme or zebrafish hindbrain, activation of the canonical Wnt signaling pathway via pharmacological inhibition of GSK $\beta$ resulted in decreased Sema3a and -3 e expression and had no effect on Sema3d in mouse calvarial osteoblasts. This indicates that there may be differential regulation of class III semaphorins downstream of canonical or noncanonical Wnt signaling and/or cell- or tissue-type differences in regulation. Our results demonstrate for the first time that at least two members of the class III semaphorins, Sema3a and $-3 e$, are transcriptionally repressed following upregulation of $\beta$-catenin signaling mouse; but the functional consequences of this remain to be elucidated.

While previous studies have focused on the effects of semaphorins on osteoclast formation and function [7, 35], we investigated the effects of recombinant Sema3e directly on osteoblasts. Since expression of PlexinD1 increased during differentiation of calvarial osteoblasts, we reasoned there may be an autocrine role for Sema3e in osteoblast formation/function. Autocrine actions of class III semaphorins have been demonstrated to regulate the survival of kidney podocytes [36]. Sema3a acts in an autocrine fashion in several tumor types, causing dispersal of glioblastoma in a Rac1-dependent manner [37], stimulating adhesion via the $\alpha_{2} \beta_{1}$ integrin in breast cancer cells [38], and regulating the angiogenic potential of endothelial cells [25]. Here, we demonstrate that Sema3e can affect osteoblasts in an autocrine fashion, inhibiting migration but not proliferation, 
resulting in a decreased reclamation of wound area in a scratch assay. Negative regulation of migration by class III semaphorins has been observed before, with Serini et al. [39] demonstrating the ability of Sema3a to inhibit the adhesion and migration of chick embryo endothelial cells due to altered activation of $\beta_{1}$ integrins. Sema3e is known to inhibit the migration of $\mathrm{CD} 9^{+}$thymocytes toward CCL25 and CCL21 chemokines in a pathway involving PlexinD1 [40]. However, furin-dependent processing of Sema3e results in a truncated p61-Sema3e protein that promotes endothelial cell migration [41], suggesting that individual semaphorins may have differential effects on migration depending on the processing of the protein. We used full-length recombinant Sema3e in our osteoblast cultures and observed a negative effect on cell migration, but we do not know if Sema3e is processed in a similar fashion to that observed by Christensen et al. [41] or whether this would alter the effects observed by cleavage to the p61-Sema3e form.

Little is known about the effects of semaphorins on bone development and homeostasis, although there is accumulating evidence of their involvement. Knockout mouse models have revealed rib-patterning and vertebral body defects in Sema3a [42] and PlexinD1 [9] knockout mice, which may be due to developmental defects in axial skeletogenesis. Sema3a signaling in rat bone both temporally and spatially coincides with infiltration of bone with blood vessels and nerve fibers [6], suggesting defective innervation and perfusion might be the cause of the defects observed in Sema3a knockout mice. That PlexinD1 mice display a skeletal phenotype might suggest an involvement of Sema3e in skeletogenesis but may also be secondary to observed vascular defects [43]. PlexinA1 knockout mice do not display the same morphological defects as Sema3a and PlexinD1 knockout mice but have an osteopetrotic phenotype due to decreased osteoclast number and activity attributable to defective Sema6d, but not Sema3a, signaling [44].

Only a recent publication by Sutton et al. [7] has demonstrated a clear role for a class III semaphorin in the direct local regulation of bone homeostasis: osteoblast-targeted expression of Sema3b-induced ostoeclastogenesis resulting in a osteopenic mouse model, while Sema3b transgenic osteoblasts displayed increased differentiation and mineralization compared to wild-type cells in vitro. Similar to Sutton et al. [7], we confirmed expression of Sema3b in osteoblasts and observed an increase in Sema3b expression in response to differentiation, although our results suggest that cell density and time in culture may be more important factors for the regulation of Sema3d and Sema3e expression than osteoblast differentiation. As Sema3a did not alter in expression during growth, the significant increase in expression in mineralizing cultures appears to be due solely to differentiation. However, no change in Sema3a expression was observed after treatment with PTH or $1,25(\mathrm{OH})_{2} \mathrm{D}_{3}$, both of which have known effects on osteoblast differentiation. We did observe an increase in Sema3e in response to $1,25(\mathrm{OH})_{2} \mathrm{D}_{3}$ treatment, as did Sutton et al. [7] for Sema3b. Sutton et al. [7] reported an increase in Sema3b expression within $3 \mathrm{~h}$ of treatment, while we did not observe any changes in Sema3e expression until much later time points. We observed no change in expression of Sema3a or $-3 \mathrm{~d}$ in response to $1,25(\mathrm{OH})_{2} \mathrm{D}_{3}$ treatment, demonstrating that some, but not all, class III semaphorins are regulated either directly (as is likely for Sema3b) or indirectly (i.e., Sema3e) by $1,25(\mathrm{OH})_{2} \mathrm{D}_{3}$. In contrast to Sutton et al. [7], we observed a negative effect of Sema3e on osteoclast formation, suggesting that Sema3e may act in opposition to Sema3b, which would be supported by the significant decrease in Sema3e expression in mineralizing osteoblasts, while Sema3b tends to decrease. This raises the possibility that Sema3e acts as a negative regulator of bone resorption during increased bone formation, while Sema3b may promote osteoclast formation during times of osteoblast quiescence. Further studies are needed to determine whether the relative expression of Sema3e and $-3 b$ (plus, indeed, other class III semaphorins) acts as a metaphorical weight on the seesaw of bone turnover, tipping the balance in favor of resorption or formation.

We have established that all, with the exception of Sema3f, class III semaphorins are expressed by osteoblasts and that they may all be differentially regulated by cell growth, differentiation, and diverse signaling pathways essential for the maintenance of bone homeostasis. Furthermore, we suggest an autorine effect on osteoblast migration and a role for Sema3e as a paracrine mediator of osteoclast formation. We observed a 10-fold increase in Sema3d expression in mineralizing cultures that correlates with increasing cell density. As Sema3d is regulated in the opposite direction from Sema3e, it would be interesting to observe if Sem3d has opposing effects on osteoclast formation compared to Sema3e. However, as Sem3d expression was unaffected by treatment with $\mathrm{PTH}$, BIO, or $1,25(\mathrm{OH})_{2} \mathrm{D}_{3}$, we did not investigate this protein further. In addition, as Sema3d would signal via the involvement of both a plexin and a neuropilin [45], we would not be able to easily rule out the involvement of antagonism with VEGF signaling on any observed effects. VEGF is known to enhance osteoclast activity and survival [46], indicating that competition between the Sema3d receptor complex and the VEGFR for neuropilins could have confounding effects on osteoclast cultures. Previous reports have suggested no effect of Sema3a on osteoclasts [44], while Sema3b appears to be a strong regulator of osteoclast formation [7]. Our data indicate that Sema3b is not the only class III semaphorin to have effects on osteoclast function 
but that it has opposing effects to those demonstrated by Sutton et al. [7] with Sema3b. This raises the possibility that not only are class III Sema proteins important in coupling osteoblast and osteoclast activity but there might also be opposing actions on bone turnover dependent on the expression of the class III Sema proteins themselves and the nuances of the receptor complexes with which they interact.

Acknowledgements The authors acknowledge the help of Christine Duncan and Hayleigh Plant during the preliminary work for this article. This work was initiated as part of the European Specific Targeted Research Project (STREP) Framework 6 consortium ANABONOS and received further funding from Arthritis Research UK (grant 17285).

Conflict of interest The authors have stated that they have no conflict of interest.

Open Access This article is distributed under the terms of the Creative Commons Attribution Noncommercial License which permits any noncommercial use, distribution, and reproduction in any medium, provided the original author(s) and source are credited.

\section{References}

1. Pasterkamp RJ, Kolodkin AL (2003) Semaphorin junction: making tracks toward neural connectivity. Curr Opin Neurobiol 13(1):79-89

2. Tamagnone L, Comoglio PM (2000) Signalling by semaphorin receptors: cell guidance and beyond. Trends Cell Biol 10(9):377-383

3. Yazdani U, Terman JR (2006) The semaphorins. Genome Biol 7(3):211

4. Gu C, Yoshida Y, Livet J, Reimert DV, Mann F, Merte J, Henderson CE, Jessell TM, Kolodkin AL, Ginty DD (2005) Semaphorin $3 \mathrm{E}$ and PlexinD1 control vascular pattern independently of neuropilins. Science 307(5707):265-268

5. Togari A, Mogi M, Arai M, Yamamoto S, Koshihara Y (2000) Expression of mRNA for axon guidance molecules, such as semaphorin-III, netrins and neurotrophins, in human osteoblasts and osteoclasts. Brain Res 878(1-2):204-209

6. Gomez C, Burt-Pichat B, Mallein-Gerin F, Merle B, Delmas PD, Skerry TM, Vico L, Malaval L, Chenu C (2005) Expression of Semaphorin-3A and its receptors in endochondral ossification: potential role in skeletal development and innervation. Dev Dyn 234(2):393-403

7. Sutton AL, Zhang X, Dowd DR, Kharode YP, Komm BS, Macdonald PN (2008) Semaphorin 3B is a 1, 25-dihydroxyvitamin D3-induced gene in osteoblasts that promotes osteoclastogenesis and induces osteopenia in mice. Mol Endocrinol 22(6):1370-1381

8. Harper J, Gerstenfeld LC, Klagsbrun M (2001) Neuropilin-1 expression in osteogenic cells: down-regulation during differentiation of osteoblasts into osteocytes. J Cell Biochem 81(1):82-92

9. Kanda T, Yoshida Y, Izu Y, Nifuji A, Ezura Y, Nakashima K, Noda M (2007) PlexinD1 deficiency induces defects in axial skeletal morphogenesis. J Cell Biochem 101(6):1329-1337

10. Christensen CR, Klingelhöfer J, Tarabykina S, Hulgaard EF, Kramerov D, Lukanidin E (1998) Transcription of a novel mouse semaphorin gene, M-semaH, correlates with the metastatic ability of mouse tumor cell lines. Cancer Res 58(6):1238-1244

11. Rozen S, Skaletsky H (2000) Primer3 on the WWW for general users and for biologist programmers. Methods Mol Biol 132:365-386

12. Coxon FP, Helfrich MH, van't Hof RJ, Sebti SM, Ralston SH, Hamilton AD, Rogers MJ (2000) Protein geranylgeranylation is required for osteoclast formation, function, and survival: inhibition by bisphosphonates and GGTI-298. J Bone Miner Res 15:1467-1476

13. van't Hof RJ, von Lindern M, Nijweide PJ, Beug H (1997) Stem cell factor stimulates chicken osteoclast activity in vitro. FASEB J 11:287-293

14. Pfaffl MW (2001) A new mathematical model for relative quantification in real-time RT-PCR. Nucleic Acids Res 29(9):e45

15. Datta NS, Abou-Samra AB (2009) PTH and PTHrP signaling in osteoblasts. Cell Signal 21(8):1245-1254

16. Tenenbaum HC, Heersche JN (1982) Differentiation of osteoblasts and formation of mineralized bone in vitro. Calcif Tissue Int 34(1):76-79

17. Skjødt H, Gallagher JA, Beresford JN, Couch M, Poser JW, Russell RG (1985) Vitamin D metabolites regulate osteocalcin synthesis and proliferation of human bone cells in vitro. J Endocrinol 105(3):391-396

18. Haneji T, Kurihara N, Ikeda K, Kumegawa M (1983) $1 \alpha$, 25-Dihydroxyvitamin D3 and analogues of vitamin D3 induce alkaline phosphatase activity in osteoblastic cells derived from newborn mouse calvaria. J Biochem 94(4):1127-1132

19. Tamura M, Nemoto E, Sato MM, Nakashima A, Shimauchi H (2010) Role of the Wnt signaling pathway in bone and tooth. Front Biosci (Elite Ed) 2:1405-1413

20. Berndt JD, Halloran MC (2006) Semaphorin 3d promotes cell proliferation and neural crest cell development downstream of TCF in the zebrafish hindbrain. Development 133(20):3983-3992

21. Kettunen P, Løes S, Furmanek T, Fjeld K, Kvinnsland IH, Behar O, Yagi T, Fujisawa H, Vainio S, Taniguchi M, Luukko K (2005) Coordination of trigeminal axon navigation and patterning with tooth organ formation: epithelial-mesenchymal interactions, and epithelial Wnt4 and Tgfbeta1 regulate semaphorin 3a expression in the dental mesenchyme. Development 132(2):323-334

22. Aberle H, Bauer A, Stappert J, Kispert A, Kemler R (1997) Betacatenin is a target for the ubiquitin-proteasome pathway. EMBO J 16(13):3797-3804

23. Sato N, Meijer L, Skaltsounis L, Greengard P, Brivanlou AH (2004) Maintenance of pluripotency in human and mouse embryonic stem cells through activation of Wnt signaling by a pharmacological GSK-3-specific inhibitor. Nat Med 10:55-63

24. Dai J, Rabie ABM (2007) VEGF: an essential mediator of both angiogenesis and endochondral ossification. J Dent Res 86:937

25. Vacca A, Scavelli C, Serini G, Di Pietro G, Cirulli T, Merchionne F, Ribatti D, Bussolino F, Guidolin D, Piaggio G, Bacigalupo A, Dammacco F (2006) Loss of inhibitory semaphorin 3A (SEMA3A) autocrine loops in bone marrow endothelial cells of patients with multiple myeloma. Blood 108(5):1661-1667

26. Klagsbrun M, Eichmann A (2005) A role for axon guidance receptors and ligands in blood vessel development and tumor angiogenesis. Cytokine Growth Factor Rev 16(4-5):535-548

27. Catalano A, Caprari P, Moretti S, Faronato M, Tamagnone L, Procopio A (2006) Semaphorin-3A is expressed by tumor cells and alters T-cell signal transduction and function. Blood 107(8):3321-3329

28. Kikutani H, Suzuki K, Kumanogoh A (2007) Immune semaphorins: increasing members and their diverse roles. Adv Immunol 93:121-143

29. Bielenberg DR, Hida Y, Shimizu A, Kaipainen A, Kreuter M, Kim CC, Klagsbrun M (2004) Semaphorin 3F, a chemorepulsant 
for endothelial cells, induces a poorly vascularized, encapsulated, nonmetastatic tumor phenotype. J Clin Invest 114(9):1260-1271

30. Karayan-Tapon L, Wager M, Guilhot J, Levillain P, Marquant C, Clarhaut J, Potiron V, Roche J (2008) Semaphorin, neuropilin and VEGF expression in glial tumours: SEMA3G, a prognostic marker? Br J Cancer 99(7):1153-1160

31. Chedotal A, Kerjan G, Moreau-Fauvarque C (2005) The brain within the tumour: new roles for axon guidance molecules in cancers. Cell Death Diff 12:1044-1056

32. Bachelder RE, Lipscomb EA, Lin X, Wendt MA, Chadborn NH, Eickholt BJ, Mercurio AM (2003) Competing autocrine pathways involving alternative neuropilin-1 ligands regulate chemotaxis of carcinoma cells. Cancer Res 63(17):5230-5233

33. Guttmann-Raviv N, Shraga-Heled N, Varshavsky A, GuimaraesSternberg C, Kessler O, Neufeld G (2007) Semaphorin-3A and semaphorin-3F work together to repel endothelial cells and to inhibit their survival by induction of apoptosis. J Biol Chem 282(36):26294-26305

34. Catalano A, Caprari P, Rodilossi S, Betta P, Castellucci M, Casazza A, Tamagnone L, Procopio A (2004) Cross-talk between vascular endothelial growth factor and semaphorin-3A pathway in the regulation of normal and malignant mesothelial cell proliferation. FASEB J 18(2):358-360

35. Zhang Y, Singh MK, Degenhardt KR, Lu MM, Bennett J, Yoshida Y, Epstein JA (2009) Tie2Cre-mediated inactivation of plexinD1 results in congenital heart, vascular and skeletal defects. Dev Biol 325(1):82-93

36. Guan F, Villegas G, Teichman J, Mundel P, Tufro A (2006) Autocrine class III semaphorin system regulates slit diaphragm proteins and podocyte survival. Kidney Int 69(9):1564-1569

37. Bagci T, Wu JK, Pfannl R, Ilag LL, Jay DG (2009) Autocrine semaphorin 3A signaling promotes glioblastoma dispersal. Oncogene 28(40):3537-3550

38. Pan H, Wanami LS, Dissanayake TR, Bachelder RE (2009) Autocrine semaphorin3A stimulates alpha2 beta1 integrin expression/function in breast tumor cells. Breast Cancer Res Treat 118(1):197-205
39. Serini G, Valdembri D, Zanivan S, Morterra G, Burkhardt C, Caccavari F, Zammataro L, Primo L, Tamagnone L, Logan M, Tessier-Lavigne $\mathrm{M}$, Taniguchi $\mathrm{M}$, Püschel AW, Bussolino $\mathrm{F}$ (2003) Class III semaphorins control vascular morphogenesis by inhibiting integrin function. Nature 424(6947):391-397

40. Choi YI, Duke-Cohan JS, Ahmed WB, Handley MA, Mann F, Epstein JA, Clayton LK, Reinherz EL (2008) PlexinD1 glycoprotein controls migration of positively selected thymocytes into the medulla. Immunity 29(6):888-898

41. Christensen C, Ambartsumian N, Gilestro G, Thomsen B, Comoglio P, Tamagnone L, Guldberg P, Lukanidin E (2005) Proteolytic processing converts the repelling signal Sema3E into an inducer of invasive growth and lung metastasis. Cancer Res 65(14):6167-6177

42. Behar O, Golden JA, Mashimo H, Schoen FJ, Fishman MC (1996) Semaphorin III is needed for normal patterning and growth of nerves, bones and heart. Nature 383(6600):525-528

43. Zhang Y, Singh MK, Degenhardt KR, Lu MM, Bennett J, Yoshida Y, Epstein JA (2009) Tie2Cre-mediated inactivation of plexinD1 results in congenital heart, vascular and skeletal defects. Dev Biol 325(1):82-93

44. Takegahara N, Takamatsu H, Toyofuku T, Tsujimura T, Okuno T, Yukawa K, Mizui M, Yamamoto M, Prasad DV, Suzuki K, Ishii M, Terai K, Moriya M, Nakatsuji Y, Sakoda S, Sato S, Akira S, Takeda K, Inui M, Takai T, Ikawa M, Okabe M, Kumanogoh A, Kikutani H (2006) PlexinA1 and its interaction with DAP12 in immune responses and bone homeostasis. Nat Cell Biol $8(6): 615-622$

45. Feiner L, Koppel AM, Kobayashi H, Raper JA (1997) Secreted chick semaphorins bind recombinant neuropilin with similar affinities but bind different subsets of neurons in situ. Neuron 19(3):539-545

46. Nakagawa M, Kaneda T, Arakawa T, Morita S, Sato T, Yomada T, Hanada K (2000) Vascular endothelial growth factor (VEGF) directly enhances osteoclastic bone resoprtion and survival of mature osteoclasts. FEBS Lett 473(2):161-164 